\title{
Factors influencing postoperative abdominal pain in DIEP flap breast reconstruction
}

\author{
Jin-Woo Park", Ik Hyun Seong", Kyong-Je Woo \\ Department of Plastic and Reconstructive Surgery, Ewha Womans University Mokdong Hospital, Ewha University College of Medicine, Seoul, \\ Korea \\ Contributions: (I) Conception and design: All authors; (II) Administrative support: JW Park, IH Seong; (III) Provision of study materials or patients: \\ KJ Woo; (IV) Collection and assembly of data: All authors; (V) Data analysis and interpretation: All authors; (VI) Manuscript writing: All authors; (VII) \\ Final approval of manuscript: All authors. \\ "These authors are co-first authors, as they contributed equally to this work. \\ Correspondence to: Kyong-Je Woo, MD, PhD. Assistant Professor, Department of Plastic and Reconstructive Surgery, College of Medicine, Ewha \\ Womans University, Ewha Womans University Mokdong Hospital, 1071, Anyangcheon-ro, Yangcheon-gu, Seoul 07985, Korea. \\ Email: economywoo@gmail.com.
}

Background Identification of a subgroup of patients with severe postoperative pain is important for adequate pain management for enhanced, fast recovery after deep inferior epigastric artery perforator (DIEP) flap breast reconstruction. The purpose of this study was to identify factors influencing postoperative abdominal pain in patients undergoing DIEP flap breast reconstruction.

Methods: Consecutive patients who underwent unilateral breast reconstruction using DIEP free flaps from October 2018 to July 2020 were included in this study. Visual analog scale (VAS)-guided postoperative pain scores were documented every 3 hours until 48 hours postoperatively. Factors affecting patient-reported pain scores were analyzed using a linear mixed-effects model. Independent variables included patient characteristics, history of previous open abdominal surgery, and operative factors including the flap size, flap weight, use of a unipedicled or bipedicled flap, number of perforators, timing of reconstruction, and use of a catheter-based subcutaneous plane block in the abdomen. A catheter was placed above the rectus fascia during closure, and analgesics were continuously infused during the 48 hours using an ON-Q Pain Relief System (I-Flow Co., Lake Forest, CA, USA).

Results: Fifty-five patients were included in the analysis. In the linear mixed effect model using multiple clinical variables, the harvested flap weight was significantly associated with the degree of pain ( $\beta$ coefficient $=0.157, \mathrm{P}=0.008)$. The pain degrees significantly decreased according to postoperative days $(\beta$ coefficient $=-0.649, \mathrm{P}<0.001)$. The flap type (unipedicle or bipedicle), number of perforators, timing of reconstruction, and history of previous abdominal surgery did not influence pain degrees. The use of subcutaneous plane block did not affect the degree of pain or dose of analgesics used.

Conclusions: A larger flap weight is associated with an increased degree of pain in patients undergoing DIEP flap breast reconstructions. Vigorous pain management might be necessary when a large flap is elevated.

Keywords! Breast reconstruction; deep inferior epigastric artery perforator flap (DIEP); pain

Submitted Mar 17, 2021. Accepted for publication May 18, 2021.

doi: $10.21037 /$ gs-21-175

View this article at: https://dx.doi.org/10.21037/gs-21-175 


\section{Introduction}

A microsurgical breast reconstruction using an abdominal flap (deep inferior epigastric artery perforator flap, DIEP) is the most common form of autologous breast reconstruction (1). A DIEP flap is associated with increased patient satisfaction with low donor site morbidity (2). However, patients often complain of severe postoperative pain at abdominal donor sites, especially during the early postoperative period. Enhanced Recovery After Surgery (ERAS) protocols have been proposed in microsurgical breast reconstruction, and postoperative pain management is an importance component in these protocols (3).

Uncontrolled postoperative pain at the abdominal donor site after DIEP flap breast reconstruction is a common complaint of the patients, and it can be associated with increased opioid consumption, prolonged hospitalizations, and chronic pain syndromes in some patients (4). For this reason, various methods have been used to reduce postoperative donor-site pain, including the systemic use of opioids, patient-controlled anesthesia (PCA), continuous anesthetic infusion via a catheter placed into the donor site, and a direct nerve block via transversus abdominis plane (TAP) blockade $(5,6)$. Despite the use of the same protocol for postoperative pain management, the degree of postoperative pain is different between individuals, and some patients complain of uncontrolled, severe pain (7). In this context, identification of a subgroup of patients with severe postoperative pain is important for adequate pain control, which improves patient satisfaction and surgical outcomes. However, little is known regarding factors influencing postoperative donor site pain in patients undergoing DIEP flap breast reconstruction. The purpose of this study was to identify factors influencing postoperative abdominal pain in patients undergoing DIEP flap breast reconstruction.

We present the following article in accordance with the STROBE reporting checklist (available at https://dx.doi. org/10.21037/gs-21-175).

\section{Methods}

\section{Population and variables}

A total of 55 patients who underwent unilateral breast reconstruction using DIEP flap at a single institution between October 2018 and July 2020 were included in this study. This study was approved by the institutional review board of our institution (IRB No. 2020-03-015), which waived the need for informed consent because the research involved no more than minimal risk to the study participants, and was conducted in accordance with the Declaration of Helsinki (as revised in 2013).

Data were prospectively collected for consecutive patients who underwent DIEP flap breast reconstruction performed by a single surgeon $(\mathrm{KJW})$. In bipedicled DIEP flap reconstructions, intra-flap anastomosis with turbocharging technique was used. We captured the following data: demographic information, history of previous open abdominal surgery, harvested flap weight, unipedicled or bipedicled flap, number of perforators, harvested flap weight, presence or absence of a catheterbased subcutaneous plane block using an ON-Q Pump (I-Flow Co., Lake Forest, CA, USA), timing of reconstruction (immediate/delayed), and dosage of pain medications.

\section{Measurement of pain scores after surgery}

The postoperative degree of pain at the donor site was measured using a visual analog scale (VAS), which has been commonly used to assess severity of pain in abdomen-based breast reconstruction, with scores ranging from 0 to 10 . The mean pain scores were calculated over time to evaluate pain score patterns. Patients were educated that a VAS score of 5 was defined as the degree of pain at which the patient found it difficult to sleep or rest without additional pain control, and a VAS score of 10 was defined as pain as severe as death. The pain scores were evaluated by an attending physician or surgeon and recorded at every scheduled postoperative flap examination. The postoperative VAS scores were documented every 3 hours until 48 postoperative hours. Thereafter, VAS scores were captured every 6 hours until the completion of 5 postoperative days.

\section{Subcutaneous plane block}

An ON-Q Pain Relief System was applied when it was available and the patient had consented to its use for a continuous percutaneous anesthetic infusion. The infusion catheter was placed above the rectus fascia for subcutaneous layer block at the time of donor-site closure. A total of $400 \mathrm{~mL}$ of an anesthetic solution consisting of $260 \mathrm{~mL}$ of $0.75 \%$ ropivacaine (AstraZeneca Korea, Ltd., Seoul, Republic of Korea) and $140 \mathrm{~mL}$ of normal saline was continuously infused for 48 hours. For patients who did not receive a percutaneous ON-Q infusion, the 
abdominal wound was closed without catheter placement.

\section{Donor-site closure procedure}

Abdominal donor-site closure was performed using a documented standard protocol. Two-layer closure of the anterior rectus sheath was performed with 1-0 Vicryl in interrupted pattern and 1-0 Prolene running sutures without mesh support. Abdominal plication of the rectus fascia was performed. Two closed suction drains were inserted into the abdomen.

\section{Postoperative analgesic use}

An intravenous (IV) PCA device administering fentanyl (Hana Pharm Co., Ltd., Seoul, Republic of Korea) and ramosetron (Daiichi Sankyo Korea Co., Ltd.) is routinely applied in the postanesthesia care unit after surgery. The patients were instructed to press the button whenever they felt that their pain control was inadequate. The IV PCA device was removed after 48 hours, at which time the remaining dose was recorded. The IV PCA device started with a total volume of $60 \mathrm{~mL}$ consisting of 10 to $12 \mu \mathrm{g}$ fentanyl per $\mathrm{kg}$ of the patient's body weight and was continuously infused at a rate of $0.5 \mathrm{~mL} / \mathrm{h}$. The device was set to purge $0.5 \mathrm{~mL}$ boluses whenever the patient pressed the designated button. When the patient required additional analgesia, pain control was augmented using IV acetaminophen, IV ketorolac, or oral acetaminophen. For comparison, pain medications were converted to morphine equivalents to calculate the total usage of pain medication.

\section{Statistical analysis}

R language version 3.3.3 (R Foundation for Statistical Computing, Vienna, Austria) and T\&F program ver. 3.0 (YooJin BioSoft, Korea) were used for all statistical analyses. Continuous variables are presented as mean \pm standard deviation or median (interquartile range) based on the distribution of the data, and frequencies and proportions are used to describe categorical variables. Linear mixed-effects models were used to analyze the effect of clinical variables on the VAS scores that were repeatedly measured until 48 hours postoperatively. The mixed-effects models included each clinical variable and a time variable as fixed effects and a random intercept and slope. The differences in morphine equivalents and morphine equivalents per body weight between the subcutaneous plane block and control groups were analyzed using the Mann-Whitney $\mathrm{U}$ test. Analysis of covariance was also performed to adjust confounding covariates.

\section{Results}

Fifty-five patients were included in the analysis. The mean age of the patients was $48.76 \pm 7.9$ years, and the median body mass index (BMI) was $23.49(21.66-25.92) \mathrm{kg} / \mathrm{m}^{2}$. Fifteen patients had a history of previous abdominal surgery. Fifty-three patients underwent unipedicled flap reconstruction, and the remaining 2 patients underwent bipedicled flap reconstruction using a turbocharging technique. The number of perforators was 1 for 8 patients, 2 for 30 patients, 3 for 14 patients, and 4 for 3 patients. All 55 patients received IV PCA. Two patients complained of nausea due to IV PCA, and the IV PCA device was removed in these patients before the 48 hours had elapsed. Subcutaneous plane block was applied in 22 patients. Fortyone patients underwent immediate reconstructions, and 14 patients underwent delayed reconstructions. The median harvest flap weight was $661(502-902) \mathrm{g}$. The median morphine equivalent was 40 (25-65) $\mathrm{mg}$ (Table 1).

The mean VAS score of the overall patients gradually decreased with time. The mean VAS scores at 3 hours and 48 hours postoperatively were $3.76 \pm 1.77$ and $2.74 \pm 1.60$, respectively. The trend in VAS scores over time is shown in Figure 1.

In the analysis of effects of time and each clinical variable on the postoperative VAS scores using linear mixed effect models, body weight $(\beta$ coefficient $=0.045, \mathrm{P}=0.009$ ), BMI ( $\beta$ coefficient $=0.130, \mathrm{P}=0.005)$, and harvested flap weight ( $\beta$ coefficient $=0.171, \mathrm{P}=0.005)$ were significantly associated with VAS scores. Age, height, history of previous abdominal surgery, bilateral pedicle harvest, number of perforators, and subcutaneous plane block use were not significantly associated with VAS scores. Time was significantly associated with VAS scores in all analytical models for each variable (Table 2). In the analysis of effects of time and multiple clinical variables on the postoperative VAS scores using linear mixed effect models, VAS scores significantly decreased with time ( $\mathrm{P}$ value $<0.001)$. An increased harvested flap weight was also significantly associated with higher VAS scores $(\mathrm{P}=0.008)$. The VAS scores of patients undergoing DIEP flap breast reconstruction decreased 0.898 points for each postoperative day during the 48-hour postoperative period and increased 0.157 points for each 100 -g increase in harvested flap weight. However, subcutaneous plane block use did not 
Table 1 Clinical and surgical variables of overall patients

\begin{tabular}{|c|c|}
\hline Variable & Value \\
\hline Age, mean $\pm S D, y$ & $48.76 \pm 7.9$ \\
\hline BMI, median (IQR), $\mathrm{kg} / \mathrm{m}^{2}$ & 23.49 (21.66-25.92) \\
\hline \multicolumn{2}{|l|}{ Prior abdominal surgery } \\
\hline Yes & $15(27.3)$ \\
\hline No & $40(72.7)$ \\
\hline Flap height, median (IQR), $\mathrm{cm}^{2}$ & $12.5(12-13)$ \\
\hline Flap width, median (IQR), $\mathrm{cm}^{2}$ & $30(28-33)$ \\
\hline Flap area, mean $\pm \mathrm{SD}, \mathrm{g}$ & $389.68 \pm 70.21$ \\
\hline \multicolumn{2}{|l|}{ Bilateral pedicle harvest } \\
\hline Yes & $2(3.6)$ \\
\hline No & $53(96.4)$ \\
\hline \multicolumn{2}{|l|}{ Perforator number } \\
\hline 1 & $8(14.5)$ \\
\hline 2 & $30(54.5)$ \\
\hline 3 & $14(25.5)$ \\
\hline 4 & $3(5.5)$ \\
\hline Harvested flap weight, median (IQR), g & $661(502-902)$ \\
\hline \multicolumn{2}{|l|}{ Subcutaneous plane block use } \\
\hline Yes & $22(40)$ \\
\hline No & $33(60)$ \\
\hline \multicolumn{2}{|l|}{ Timing of reconstruction } \\
\hline Immediate & $41(74.5)$ \\
\hline Delayed & $14(25.5)$ \\
\hline Morphine equivalents, median (IQR), mg & $40(25-65)$ \\
\hline
\end{tabular}

$\mathrm{SD}$, standard deviation; BMI, body mass index; IQR, interquartile range.

have a significant effect on VAS scores $(\beta$ coefficient $=0.540$, $\mathrm{P}=0.110)$ (Table 3).

In the analysis of the difference of in morphine equivalents between the subcutaneous plane block and control groups, no significant differences in morphine equivalents or morphine equivalents per body weight were observed between the two groups $(\mathrm{P}=0.557$ and 0.751 , respectively, Table 4). After controlling for covariates including age, history of previous abdominal surgery, harvested flap weight, perforator position, and timing of reconstruction, there were also no significant differences in morphine equivalents or morphine equivalents per body weight between the two groups $(\mathrm{P}=0.871$ and 0.671 , respectively, Table 5).

\section{Discussion}

In this retrospective study, we evaluated factors associated with donor-site pain after DIEP flap breast reconstruction and demonstrated that the postoperative time and harvested flap weight were significantly associated with pain scores. The VAS scores of donor-site pain gradually decreased over time during the 48-hour postoperative period and were significantly higher when the harvested flap weight was increased. However, the use of subcutaneous plane block did not significantly affect VAS scores.

Several studies have been conducted to evaluate the effect of regional blocks on donor-site pain after abdomenbased breast reconstruction. TAP blockade is a the most widely used peripheral block that affects the nerves of the anterior abdominal wall, and its effects on reducing the length of hospital stay and opioid consumption have been well established (8-10). In a study of patients undergoing DIEP flap breast reconstruction, a bupivacaine TAP blockade was associated with significantly reduced narcotic consumption compared with infusion pump anesthetic administration and local anesthetic injection (11). Recently, liposomal bupivacaine, which has a long duration of effect, has been widely used in TAP blocks (12). The TAP block technique using liposomal bupivacaine has also been reported to reduce the length of hospital stay after surgery and costs associated with postoperative management after breast reconstruction surgery (8). Some surgeons also used a subcutaneous plane block combined with TAP blockade $(3,13)$. However, to our knowledge, the effect of a subcutaneous plane block has not been evaluated separately from the effect of TAP blockade in abdomenbased breast reconstruction. Therefore, we evaluated the effect of a subcutaneous layer block without TAP blockade using an On-Q pump after DIEP flap breast reconstruction and demonstrated that the use of an On-Q pump for the subcutaneous plane block did not significantly affect VAS scores or morphine equivalents during the 48-hour postoperative period. A subcutaneous plane block may not be recommended when performing TAP blockade in patients undergoing breast reconstruction according to the results of this study. Further comparative studies between a TAP blockade alone group and a TAP blockade combined with subcutaneous plane block group would be necessary to 


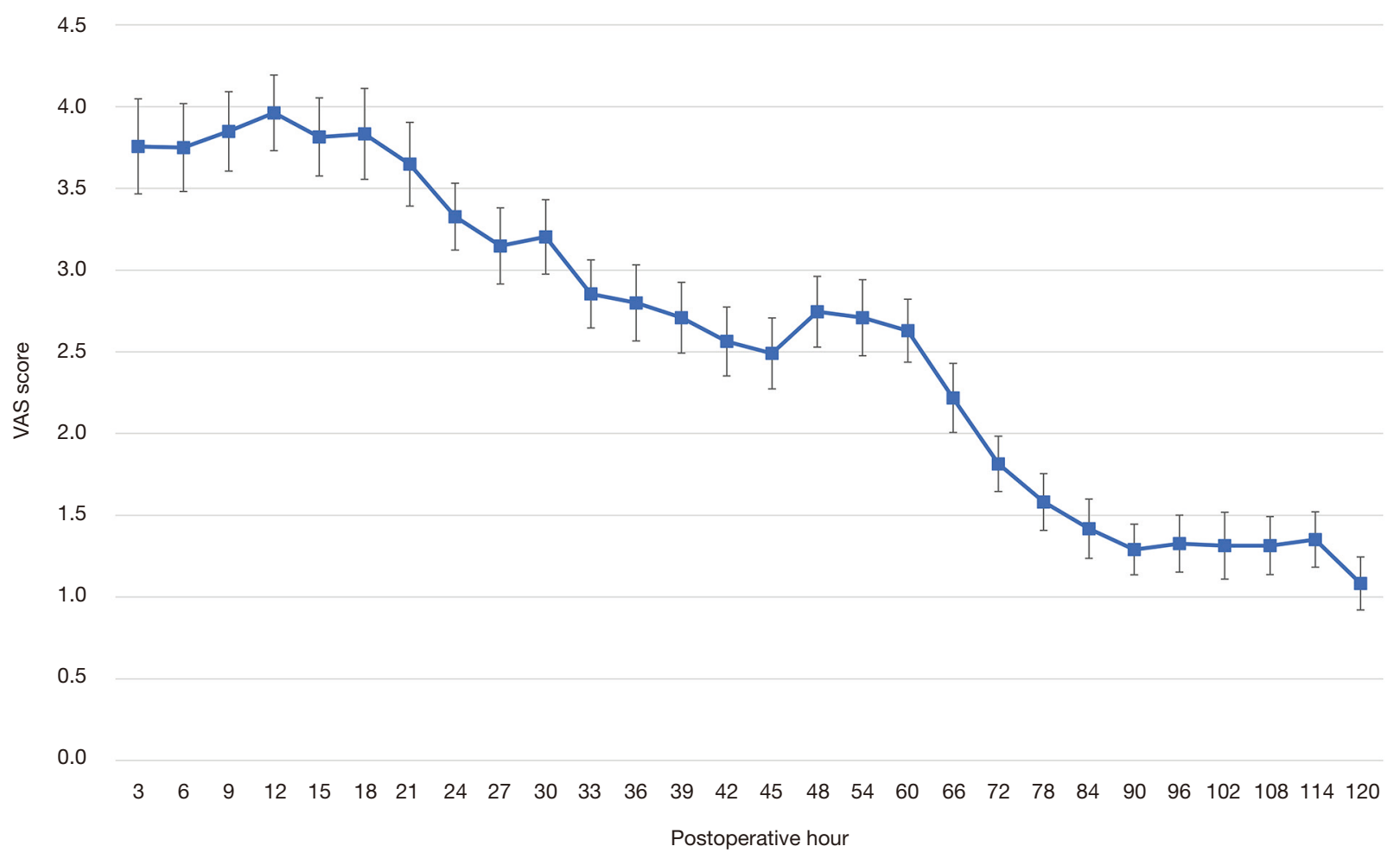

Figure 1 The VAS scores of donor-site pain after DIEP flap breast reconstruction during 5 days postoperatively are depicted using the means and standard errors of the VAS scores. VAS, visual analog scale; DIEP, deep inferior epigastric artery perforator.

confirm the results of this study.

Previous studies have demonstrated that bilateral abdomen-based breast reconstruction might lead to greater postoperative pain than unilateral reconstruction (14-16). However, the effect of bilateral harvesting of vascular pedicles on postoperative pain remains unclear because performing surgery on both breasts can also be associated with increased postoperative pain. According to a recent study by Azizi et al., morphine usage was significantly lower in a bipedicled DIEP flap group than in a unipedicled DIEP flap group in patients undergoing unilateral breast reconstruction (17). In their multivariate analysis, bipedicled reconstruction was still an independent predictor of lower immediate postoperative morphine requirements. The authors suggested that this was an unexpected finding and was in contrast to the results of previous studies and their hypothesis. According to the results of the current study, an increased flap weight was significantly associated with increased postoperative pain scores. In unilateral breast reconstruction using a DIEP flap, a bipedicled DIEP flap is used when the abdominal flap volume is relatively small.
Furthermore, a smaller harvested flap size can be achieved when using a bipedicled flap than when using a unipedicled flap because nearly the full size of the harvested flap can be used for flap inset in bipedicled reconstruction. Thus, the reduced flap weight in bipedicled reconstruction could lead to reduced postoperative pain although the pedicles were harvested bilaterally. The length of intramuscular dissection during the flap harvest, on the other hand, might not influence postoperative abdominal pain. In this study, bipedicled flap harvest or increased number of perforators of the DIEP flap did not increase postoperative abdominal pain (Table 2).

The current study is the first to demonstrate that the abdominal flap weight is a significant predictor of increased postoperative pain scores. Because the abdominal flap weight has been known to be significantly correlated with BMI, patients with a higher BMI may have more severe postoperative pain (18). Notably, there have been reports showing that TAP blockade is more effective in patients with a higher BMI. Hunter et al. reported that patients with a BMI greater than $30 \mathrm{~kg} / \mathrm{m}^{2}$ benefitted more from TAP 
Table 2 Fixed effects of time and each clinical variable on the postoperative VAS scores in the linear mixed effect models

\begin{tabular}{|c|c|c|c|c|c|}
\hline Variable & Effect & $\beta$ coefficient & Standard error & $P$ value & Semi-partial $R^{2}$ (95\% Cls) \\
\hline Age & Variable & -0.018 & 0.023 & 0.428 & $0.008(0.000-0.026)$ \\
\hline \multirow[t]{2}{*}{ Height } & Time & -0.895 & 0.126 & $<0.001$ & $0.096(0.059-0.139)$ \\
\hline & Variable & -0.005 & 0.031 & 0.874 & $0.000(0.000-0.009)$ \\
\hline Body weight & Variable & 0.045 & 0.017 & 0.009 & $0.082(0.048-0.124)$ \\
\hline \multirow[t]{2}{*}{ BMI } & Time & -0.896 & 0.126 & $<0.001$ & $0.103(0.065-0.148)$ \\
\hline & Variable & 0.130 & 0.044 & 0.005 & $0.094(0.058-0.138)$ \\
\hline History of previous abdominal surgery & Time & -0.896 & 0.126 & $<0.001$ & $0.097(0.060-0.140)$ \\
\hline Bilateral pedicle harvest & 1 vs. 0 & -0.986 & 0.933 & 0.295 & $0.015(0.002-0.037)$ \\
\hline \multirow[t]{4}{*}{ Perforator number } & Time & -0.895 & 0.126 & $<0.001$ & $0.104(0.066-0.148)$ \\
\hline & 2 vs. 1 & -0.416 & 0.485 & 0.394 & $0.010(0.001-0.029)$ \\
\hline & 3 vs. 1 & 0.711 & 0.539 & 0.193 & $0.009(0.000-0.027)$ \\
\hline & 4 vs. 1 & -0.740 & 0.822 & 0.372 & $0.020(0.005-0.046)$ \\
\hline \multirow[t]{2}{*}{ Harvested flap weight* } & Time & -0.895 & 0.126 & $<0.001$ & $0.103(0.065-0.148)$ \\
\hline & 1 vs. 0 & 0.171 & 0.058 & 0.005 & $0.096(0.059-0.140)$ \\
\hline Subcutaneous plane block use & Time & -0.898 & 0.126 & $<0.001$ & $0.100(0.062-0.144)$ \\
\hline
\end{tabular}

*, values divided by 100 were used for this analysis. VAS, visual analogue scale; Cl; confidence interval; BMI, body mass index.

Table 3 Fixed effects of time and multiple clinical variables on the postoperative VAS scores in the linear mixed effect models

\begin{tabular}{lcccr}
\hline Variable & $\beta$ coefficient & Standard error & P value & Semi-partial R $^{2}$ (95\% Cls) \\
\hline Time & -0.898 & 0.126 & $<0.001$ & $0.106(0.068-0.151)$ \\
Harvested flap weight & 0.157 & 0.057 & 0.008 & $0.083(0.048-0.124)$ \\
Subcutaneous plane block use & 0.540 & 0.332 & 0.110 & $0.030(0.010-0.059)$ \\
\hline
\end{tabular}

*, values divided by 100 were used for this analysis. VAS, visual analogue scale; Cl; confidence interval.

blockade (19). Similarly, Salibian et al. reported that patients with a BMI greater than $25 \mathrm{~kg} / \mathrm{m}^{2}$ had significant decreases in the degree of pain and opioid consumption with TAP blockade, but the same was not found in patients with a BMI less than $25 \mathrm{~kg} / \mathrm{m}^{2}$ (9). From the results of our study, it might be inferred that patients with a higher BMI could have more severe pain; thus, TAP blockade was more effective.

According to the results of this study, more vigorous pain management can be planned for patients undergoing reconstruction of large breasts or in patients in whom 
Table 4 Comparison of morphine equivalents between subcutaneous plane block and control groups

\begin{tabular}{|c|c|c|c|}
\hline Variable & Subcutaneous plane block group $(\mathrm{N}=33,60 \%)$ & Control group ( $\mathrm{N}=22,40 \%)$ & $P$ value \\
\hline $\begin{array}{l}\text { Morphine equivalents/body weight, } \\
\text { median (IQR), mg/kg }\end{array}$ & $0.60(0.47-1.07)$ & $0.89(0.44-1.11)$ & 0.751 \\
\hline
\end{tabular}

IQR, interquartile range.

Table 5 Comparison of morphine equivalents between subcutaneous plane block and control groups after adjusting covariates

\begin{tabular}{|c|c|c|c|}
\hline Variable & Subcutaneous plane block group ( $N=33,60 \%)$ & Control group ( $\mathrm{N}=22,40 \%)$ & $P$ value \\
\hline $\begin{array}{l}\text { Morphine equivalents/body weight, } \\
\text { mean } \pm \mathrm{SD}, \mathrm{mg} / \mathrm{kg}\end{array}$ & $0.85 \pm 0.15$ & $0.89 \pm 0.17$ & 0.671 \\
\hline
\end{tabular}

$\mathrm{SD}$, standard deviation.

a large flap is necessary. The information regarding factors related to postoperative pain also can be useful for preoperative patient counselling because postoperative pain is one of the most frequently identified reasons for which patients decline to undergo breast reconstruction after breast cancer surgery (20). In some cases, bipedicled flap harvesting can be actively considered when indicated, because the bipedicled flap not only can minimize tension on the abdominal donor site but also reduce donor site pain by decreasing the flap size.

This study has some limitations. First, the VAS score is a subjective pain score that is perceived by the patient. To minimize subjectivity, all of the pain scores included in this study were collected by an attending physician or surgeon in a uniform manner. Second, the sample size in this study was relatively small and patients who receiving catheter-based anesthetic infusions were not randomly selected. However, only patients who underwent breast reconstruction performed by a single surgeon were included in this study to minimize bias. Because pain severity can be affected by the timing of the examination, VAS scores were frequently evaluated by the doctors within 3-hour intervals. Finally, only subcutaneous layer placement of a catheter-based analgesic infusion pump was performed in this study. Comparative studies evaluating TAP blockade, subcutaneous layer blocks, and combined blocks are warranted to establish the most effective procedure.

\section{Conclusions}

A larger flap weight is associated with an increased degree of pain in patients undergoing DIEP flap breast reconstruction. Vigorous pain management might be necessary when a large flap is elevated, or bipedicled flap harvesting can be considered to reduce the harvested flap weight.

\section{Acknowledgments}

Funding: None.

\section{Footnote}

Reporting Checklist: The authors have completed the STROBE reporting checklist. Available at https://dx.doi. org/10.21037/gs-21-175

Data Sharing Statement: Available at https://dx.doi. org/10.21037/gs-21-175

Peer Review File: Available at https://dx.doi.org/10.21037/ gs-21-175

Conflicts of Interest: All authors have completed the ICMJE uniform disclosure form (available at https://dx.doi. org/10.21037/gs-21-175). The authors have no conflicts of interest to declare.

Etbical Statement: The authors are accountable for all aspects of the work in ensuring that questions related to the accuracy or integrity of any part of the work are appropriately investigated and resolved. The study was 
approved by the Institutional Review Board of Ewha Womans University Mokdong Hospital (IRB No. 202003-015) and performed in accordance with the principles of the Declaration of Helsinki (as revised in 2013). Individual consent for this retrospective analysis was waived.

Open Access Statement: This is an Open Access article distributed in accordance with the Creative Commons Attribution-NonCommercial-NoDerivs 4.0 International License (CC BY-NC-ND 4.0), which permits the noncommercial replication and distribution of the article with the strict proviso that no changes or edits are made and the original work is properly cited (including links to both the formal publication through the relevant DOI and the license). See: https://creativecommons.org/licenses/by-nc-nd/4.0/.

\section{References}

1. Panchal H, Matros E. Current Trends in Postmastectomy Breast Reconstruction. Plast Reconstr Surg 2017;140:7S-13S.

2. Niddam J, Bosc R, Lange F, et al. DIEP flap for breast reconstruction: retrospective evaluation of patient satisfaction on abdominal results. J Plast Reconstr Aesthet Surg 2014;67:789-96.

3. Batdorf NJ, Lemaine V, Lovely JK, et al. Enhanced recovery after surgery in microvascular breast reconstruction. J Plast Reconstr Aesthet Surg 2015;68:395402.

4. Nelson JA, Fischer JP, Pasick C, et al. Chronic pain following abdominal free flap breast reconstruction: a prospective pilot analysis. Ann Plast Surg 2013;71:278-82.

5. Rawlani V, Kryger ZB, Lu L, et al. A local anesthetic pump reduces postoperative pain and narcotic and antiemetic use in breast reconstruction surgery: a randomized controlled trial. Plast Reconstr Surg 2008;122:39-52.

6. Zhong T, Ojha M, Bagher S, et al. Transversus abdominis plane block following abdominally based breast reconstruction: study protocol for a randomized controlled trial. Trials 2013;14:424.

7. Bar-Meir ED, Yueh JH, Hess PE, et al. Postoperative Pain Management in DIEP Flap Breast Reconstruction: Identification of Patients With Poor Pain Control. Eplasty 2010;10:e59.

8. Jablonka EM, Lamelas AM, Kim JN, et al. Transversus Abdominis Plane Blocks with Single-Dose Liposomal Bupivacaine in Conjunction with a Nonnarcotic Pain Regimen Help Reduce Length of Stay following
Abdominally Based Microsurgical Breast Reconstruction. Plast Reconstr Surg 2017;140:240-51.

9. Salibian AA, Frey JD, Thanik VD, et al. Transversus Abdominis Plane Blocks in Microsurgical Breast Reconstruction: Analysis of Pain, Narcotic Consumption, Length of Stay, and Cost. Plast Reconstr Surg 2018;142:252e-263e.

10. Zhong T, Ojha M, Bagher S, et al. Transversus abdominis plane block reduces morphine consumption in the early postoperative period following microsurgical abdominal tissue breast reconstruction: a double-blind, placebocontrolled, randomized trial. Plast Reconstr Surg 2014;134:870-8.

11. Knackstedt R, Gatherwright J, Ghaznavi A, et al. Comparison of local anesthetic infusion pump bupivacaine versus transversus abdominis plane (TAP) block liposomal bupivacaine for pain management after bilateral deep inferior epigastric perforator free flap reconstruction. J Plast Reconstr Aesthet Surg 2017;70:1779-81.

12. Abdou SA, Daar DA, Wilson SC, et al. Transversus Abdominis Plane Blocks in Microsurgical Breast Reconstruction: A Systematic Review and Meta-analysis. J Reconstr Microsurg 2020;36:353-61.

13. Boehmler JH, Venturi ML, Nahabedian MY. Decreased narcotic use with an implantable local anesthetic catheter after deep inferior epigastric perforator flap breast reconstruction. Ann Plast Surg 2009;62:618-20.

14. Gassman AA, Yoon AP, Maxhimer JB, et al. Comparison of postoperative pain control in autologous abdominal free flap versus implant-based breast reconstructions. Plast Reconstr Surg 2015;135:356-67.

15. Uda H, Tomioka YK, Sarukawa S, et al. Abdominal morbidity after single- versus double-pedicled deep inferior epigastric perforator flap use. J Plast Reconstr Aesthet Surg 2016;69:1178-83.

16. Kulkarni AR, Pusic AL, Hamill JB, et al. Factors Associated with Acute Postoperative Pain Following Breast Reconstruction. JPRAS Open 2017;11:1-13.

17. Azizi AA, Mohan AT, Tomouk T, et al. Does surgical procedure type impact postoperative pain and recovery in deep inferior epigastric artery perforator flap breast reconstruction? Arch Plast Surg 2020;47:324-32.

18. Woo KJ, Kim EJ, Lee KT, et al. A Novel Method to Estimate the Weight of the DIEP Flap in Breast Reconstruction: DIEP-W, a Simple Calculation Formula Using Paraumbilical Flap Thickness. J Reconstr Microsurg 2016;32:520-7.

19. Hunter C, Shakir A, Momeni A, et al. Transversus 
Abdominis Plane Block and Free Flap Abdominal Tissue Breast Reconstruction: Is There a True Reduction in Postoperative Narcotic Use? Ann Plast Surg 2017;78:254-9.

Cite this article as: Park JW, Seong IH, Woo KJ. Factors influencing postoperative abdominal pain in DIEP flap breast reconstruction. Gland Surg 2021;10(7):2211-2219. doi: 10.21037/ gs-21-175
20. Zieliński T, Lorenc-Podgorska K, Antoszewski B. Why women who have mastectomy decide not to have breast reconstruction? Pol Przegl Chir 2015;86:451-5. 